\title{
Nova espécie de Euschistus (Mitripus) da Argentina e sul do Brasil (Hemiptera, Pentatomidae, Pentatominae) ${ }^{1}$
}

\author{
Paulo Roberto S. Bunde ${ }^{2}$ Jocélia Grazia ${ }^{2,3}$ \& Milton de S. Mendonça Junior ${ }^{2}$ \\ 1. Contribuição n ${ }^{0} 489$ do Departamento de Zoologia, Universidade Federal do Rio Grande do Sul. \\ 2. Programa de Pós-Graduação em Biologia Animal, Departamento de Zoologia, Instituto de Biociências, Universidade Federal do Rio \\ Grande do Sul, Av. Bento Gonçalves, 9500, Bloco IV, Prédio 43435, 91501-970, Porto Alegre, RS, Brasil. (paulobunde@ig.com.br; \\ jocelia@ufrgs.br; milton.mendonca@ig.com.br) \\ 3. Bolsista CNPq.
}

\begin{abstract}
New species of Euschistus (Mitripus) from Argentina and southern Brazil (Hemiptera, Pentatomidae, Pentatominae). A new species of Euschistus Dallas, 1851, E. (M.) irroratus sp. nov. from Rio Grande do Sul, Brazil and Misiones, Argentina, is described and illustrated.
\end{abstract}

KEYWORDS. Heteroptera, Pentatomini, Neotropical, new taxon, taxonomy.

RESUMO. Uma nova espécie de Euschistus Dallas, 1851, E. (M.) irroratus sp. nov. do Rio Grande do Sul, Brasil e Misiones, Argentina, é descrita e ilustrada.

PALAVRAS-CHAVE. Heteroptera, Pentatomini, Neotropical, novo táxon, taxonomia.

O subgênero Euschistus (Mitripus) foi proposto por Rolston (1978) para englobar sete espécies sulamericanas - E. (M.) acutus Dallas, 1851; E. (M.) anticus Stål, 1860; E. (M.) convergens (Herrich-Schaeffer, 1842); E. (M.) grandis Rolston, 1978; E. (M.) latus Dallas, 1852; E. (M.) legionarius Breddin, 1914 e E. (M.) tauricornis Stål, 1872. Posteriormente, mais duas espécies foram descritas por Grazia (1987a): E. (M.) hansi e E. (M.) paranticus; nesse trabalho foi designado o lectótipo macho para E. anticus Stål, 1860. GraZIA (1987b) designou o lectótipo macho e paralectótipos macho e duas fêmeas para E. legionarius Breddin, 1914, fornecendo ilustrações da genitália.

Das nove espécies que hoje compõem o subgênero, seis têm distribuição predominante no norte da Argentina, Uruguai e sul do Brasil. Euschistus (M.) grandis e E. (M.) latus ocorrem no componente Sudeste da Região Neotropical (AMORim \& Pires, 1996), em altitudes que variam de 800 a $1.700 \mathrm{~m}$ na Serra da Bocaina, Itatiaia e Parque Nacional da Serra dos Órgãos (RJ), Campos do Jordão e Campinas (SP), Rio Vermelho (SC) e São Francisco de Paula (RS). Euschistus (M.) acutus é a única espécie deste grupo com ampla distribuição no componente Caribenho e Noroeste da Região Neotropical (AMORIM \& Pires, 1996), tendo sido coletada em Trinidad, Venezuela, norte do Brasil (AM, PA), Peru e Bolívia.

$\mathrm{Na}$ Serra do Sudeste, município de Canguçu (RS, Brasil), foram coletados sete exemplares de Euschistus que não se enquadraram em nenhuma das descrições de espécies conhecidas para o Brasil. Na coleção do Departamento de Zoologia, Universidade Federal do Rio Grande do Sul (DZRS), havia mais dois exemplares procedentes da Argentina (Misiones) e um do Brasil (Pelotas, RS) conspecíficos ao material de Canguçu. Desta forma, uma nova espécie de Euschistus é aqui proposta.

\section{MATERIAL E MÉTODOS}

A terminologia adotada para as estruturas da genitália segue DuPuis (1970) e GraZia (1987a). Os exemplares foram dissecados e tratados com KOH $10 \%$ a frio. As ilustrações foram realizadas com auxílio de câmaraclara e as medidas estão expressas em milímetros. Os parâmetros morfométricos utilizados foram: comprimento da cabeça, pronoto, escutelo e total do corpo; largura da cabeça, pronoto com e sem espinho, escutelo, abdome e distância interocular (GrAZIA, 1987a). O material estudado está depositado nas seguintes instituições: DZRS, Departamento de Zoologia, Universidade Federal do Rio Grande do Sul, Porto Alegre; MACN, Museo Argentino de Ciencias Naturales Bernardino Rivadavia, Buenos Aires, Argentina; MCNZ, Museu de Ciências Naturais, Fundação Zoobotânica do Rio Grande do Sul, Porto Alegre; MECB, Museu Entomológico Ceslau Biezanko, Pelotas, Rio Grande do Sul; NMNH, National Museum of Natural History, Washington, Estados Unidos.

\section{Euschistus (Mitripus) irroratus sp. nov. (Figs. 1-10)}

Etimologia. Latim, irrorare $=$ orvalhar; alusivo ao aspecto "orvalhado" da face dorsal.

Distribuição. Brasil (Rio Grande do Sul), Argentina (Misiones).

Material-tipo. Holótipo ơ', BRASIL, Rio Grande do Sul Canguçu (Rincão da Ronda), 16.X.2003, P. Bunde \& J. Cabeleira col. (MCNZ). Parátipos. Mesma procedência do holótipo, 4 ơ', \&, 16.X. 2003, P. Bunde \& J. Cabeleira col. (DZRS); O 07.II.2003, P. Bunde \& C. Schwertner col. (DZRS); Pelotas, , 25.X.1976, E. Bassan col. (MECB). ARGENTINA, Misiones: $\sigma^{7}$, 04.II.1942, H. L. Parker (SAP Lab 647 LotNo 42-7933 - NMNH); O', s/ data (12049 - MACN). 
Descrição. Macho. Medidas: comprimento da cabeça $0,75(0,69-0,84)$; largura da cabeça 1,67 (1,53-1,69); distância interocular 1,17 (1,15-1,23); comprimento do pronoto $1,52(1,38-1,69)$, largura do pronoto $3,43(3,30$ $3,46)$; largura do pronoto com espinho $4,70(4,61-5,15)$; comprimento do escutelo 2,61 (2,53-2,69); largura do escutelo 2,76 (2,56-2,92); largura abdominal 4,40 (4,234,53); comprimento total 6,29 (6,15-6,53).

Antenas castanho-amareladas. Face dorsal castanho-clara e densamente pontuada. Cabeça, metade anterior do pronoto, ângulos umerais e quarto basal da margem costal do cório negros a negro-ferrugíneos. Ângulos umerais do pronoto pouco desenvolvidos e truncados na extremidade (Figs. 1, 2). Cicatrizes do pronoto lisas, imaculadas. Base do escutelo com duas áreas arredondadas com pontuação negra. Conexivo com pontuação negra nos ângulos anteriores e posteriores de cada segmento. Restante da pontuação da face dorsal castanho-clara. Face ventral amarelada, com densa pontuação concolor. Pernas amareladas, com pontuação castanho-escura; terço distal das tíbias e tarsos amareloferrugíneos.

Genitália. Pigóforo com o bordo ventral escavado em "U" amplamente aberto, com terço mediano bi-sinuado (Figs. 3, 5). Taça genital com duas carenas laminares menos desenvolvidas que em $E$. (M.) paranticus, sendo que apenas as carenas dorsais são visíveis dorsalmente no pigóforo (Fig. 3). Parâmeros em forma de foice, com a região do pé mais alargada do que em $E$. (M.) hansi e

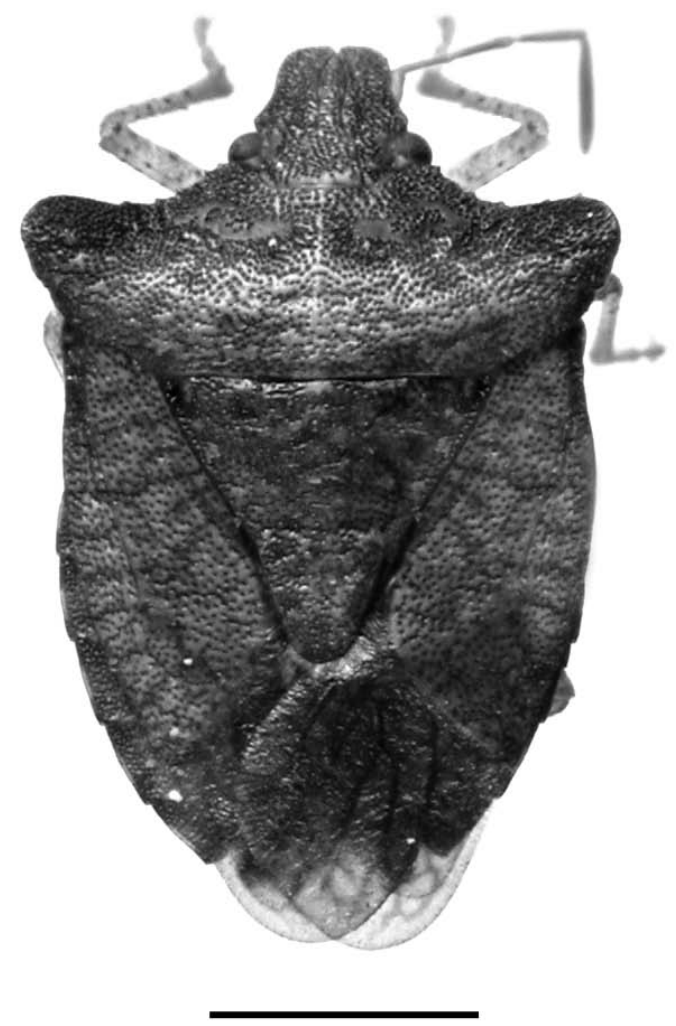

Fig. 1. Euschistus (Mitripus) irroratus, sp. nov.: holótipo macho, vista dorsal. Escala: $2 \mathrm{~mm}$.
E. (M) paranticus (Fig. 4). Phallus: conjuntiva ampla, com dois pares de processos lobados, um ventral e outro dorsal, encobrindo totalmente os processos da phallotheca e o ductus seminis distalis (Figs. 6-8)

Fêmea. Medidas: comprimento da cabeça $0,84(0,76-$ 0,92); largura da cabeça 1,80 (1,76-1,84); distância interocular 1,22 (1,15-1,30); comprimento do pronoto 1,80 $(1,69-1,92)$, largura do pronoto $3,76(3,69-3,84)$; largura do pronoto com espinho $5,30(5,23-5,38)$ comprimento do escutelo 2,88 (2,76-3,00); largura do escutelo 3,07 (3,07$3,07)$; largura abdominal $4,53(4,46-4,61)$ comprimento total $6,84(6,76-6,92)$

Genitália. Gonocoxitos 8 com os bordos pósterolaterais côncavos sobre os laterotergitos 9 , contínuos com o bordo posterior, diferindo de $E$. (M.) hansi e $E$. (M.) paranticus, que apresentam bordo posterior truncado (Fig. 9). Capsula seminalis dilatada no terço basal e digitiforme nos $2 / 3$ apicais, mais longa que a pars intermedialis (Fig. 10).

Planta-hospedeira. Os exemplares procedentes de Canguçu, cinco machos e uma fêmea, foram coletados nos meses de fevereiro e outubro de 2003, na borda das matas de galeria, sobre Schinus polygamus (Cav.) (Anacardiaceae).

Comentários. Euschistus (M.) irroratus sp. nov. se assemelha a $E$. (M.) hansi Grazia, 1987 e a $E$. (M.) paranticus Grazia, 1987, das quais se distingue principalmente pela forma dos ângulos umerais do pronoto, pouco desenvolvidos e truncados na extremidade. Em E. (M.) hansi os ângulos umerais são desenvolvidos e projetados ântero-lateralmente, enquanto que em E. (M.) paranticus são pouco desenvolvidos e arredondados no ápice. Nas fêmeas, Euschistus (M.) irroratus se distingue pela forma dos gonocoxitos 8 e nos machos, pelo terço mediano da escavação do bordo ventral do pigóforo bi-sinuado e pela forma dos parâmeros e dos processos da taça genital.

Agradecimentos. Aos curadores das coleções do MACN (Dr. A. Bachmann), MECB (Dr. Eduardo Ely e Silva) e do NMNH (Dr. T. Henry), pelo empréstimo do material. Ao CNPq, pelo financiamento do projeto, Edital Universal 1/2001 (Proc. 478787/2001-4).

\section{REFERÊNCIAS BIBLIOGRÁFICAS}

Amorim, D. S. \& Pires, M. R. S. 1996. Neotropical Biogeography and a method for maximum biodiversity estimation. In: BICUDo, C. E. M. \& Menezes, N. A. eds. Biodiversity in Brazil, a first approach. São Paulo, CNPq. p.183-219.

Dupuis, C. 1970. Heteroptera. In: Tuxen, S. L. ed. Taxonomist's glossary of genitalia of insects. Copenhagen, Munskgaard. p. 190-208

GrAZIA, J. 1987a. Duas novas espécies de Euschistus do subgênero Mitripus Rolston, 1978 (Heteroptera, Pentatomidae, Pentatomini). Revista Brasileira de Entomologia 31(1):83-88.

1987b. On some types of Heteroptera Pentatomidae preserved in the M.N.H.N., Paris. Revue Française de Entomologie, N. S., 9(1):43-46.

Rolston, L. H. 1978. A new subgenus of Euschistus (Hemiptera: Pentatomidae). Journal of the New York Entomological Society 86(2): 102-120. 

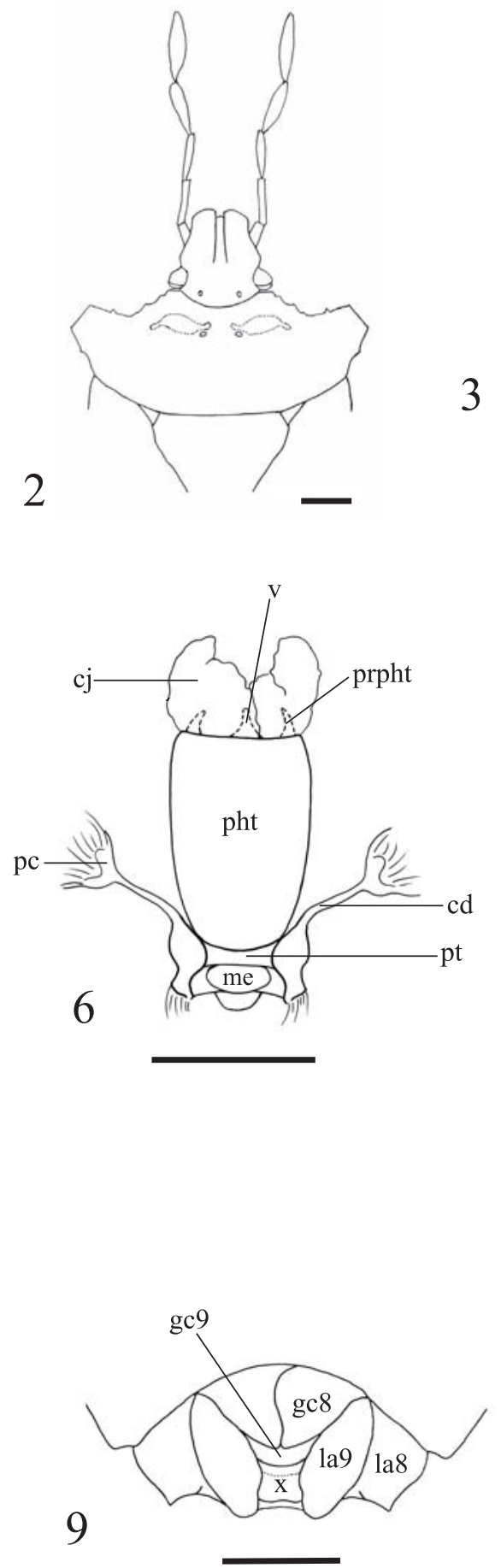
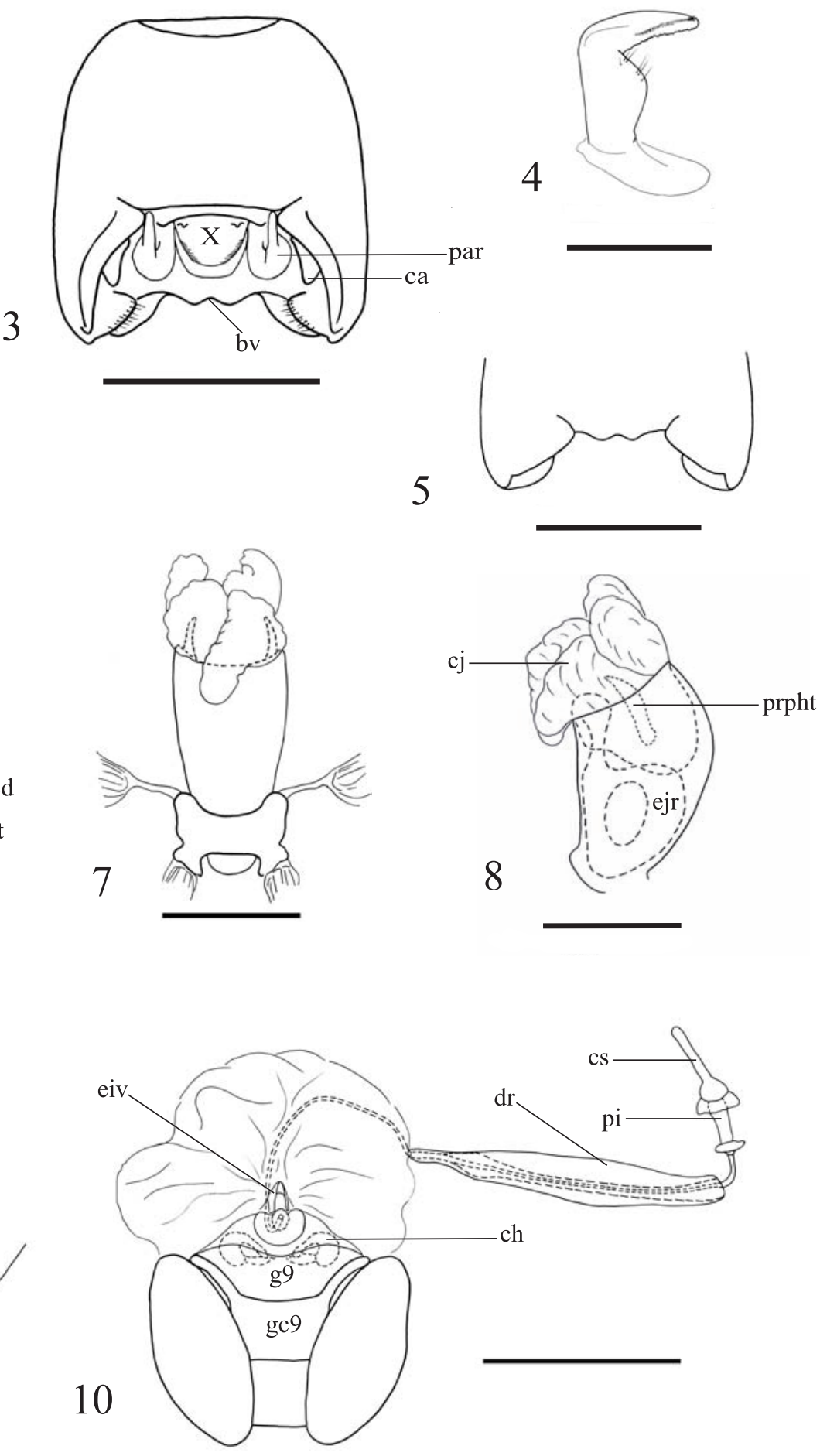

Figs. 2-10. Euschistus irroratus sp. nov.: 2, cabeça e pronoto, vista dorsal; 3, pigóforo, dorsal; 4, parâmero direito, vista lateral externa; 5, pigóforo, ventral; 6-8, phallus, respectivamente ventral, dorsal, lateral; 9, placas genitais; 10, laterotergitos 9, gonocoxitos 9, gonapófises 9 e vias genitais ectodérmicas (bv, bordo ventral; ca, carena; cd, conetivo dorsal; ch, chitinellipsen; cj, conjuntiva; cs, capsula seminalis; dr, ductus receptaculi; eiv, espessamento da intima vaginal; ejr, ejaculatory reservoir; g9, gonapófises 9; gc8, gonocoxito 8; gc9, gonocoxitos 9; la8, laterotergito 8; la9, laterotergito 9; me, membramblase; par, parâmero; pc, processus capitati; pht, phallotheca; pi, pars intermedialis; prpht, processus phallothecae; pt, ponticulus transversalis; v, vésica; $\mathrm{x}$, décimo segmento). Escalas: Figs. 2, 3, 5, 9, 10, $1 \mathrm{~mm}$; Figs. 4, 6-8, 0,5 mm. 